\title{
Bayesian modeling of item heterogeneity in dichotomous recognition memory data and prospects for computerized adaptive testing
}

\author{
Jeremie Güsten*1,2, David Berron ${ }^{1,3}$, Emrah Düzel ${ }^{\dagger 1,2,4}$, and \\ Gabriel Ziegler ${ }^{\dagger 1,2}$ \\ ${ }^{1}$ German Center for Neurodegenerative Diseases, Magdeburg, \\ Germany \\ ${ }^{2}$ Institute of Cognitive Neurology and Dementia Research, \\ Otto-von-Guericke University, Magdeburg, Germany \\ ${ }^{3}$ Clinical Memory Research Unit, Department of Clinical Sciences \\ Malmö, Lund University, Lund, Sweden \\ ${ }^{4}$ Institute of Cognitive Neuroscience, University College London, \\ London, United Kingdom
}

March 9, 2021

\footnotetext{
*Corresponding author: jeremie.guesten@med.ovgu.de

${ }^{\dagger}$ Authors contributed equally.
} 


\begin{abstract}
Most current models of recognition memory fail to separately model item and person heterogeneity which makes it difficult to assess ability at the latent construct level and prevents the administration of adaptive tests. Here we propose to employ a General Condorcet Model for Recognition (GCMR) in order to estimate ability, response bias and item difficulty in dichotomous recognition memory tasks. Using a Bayesian modeling framework and MCMC inference, we perform 3 separate validation studies comparing GCMR to the Rasch model from IRT and the 2-High-Threshold (2HT) recognition model. First, two simulations demonstrate that recovery of GCMR ability estimates with varying sparsity and test difficulty is more robust and that estimates improve from the two other models under common test scenarios. Then, using a real dataset, face validity is confirmed by replicating previous findings of general and domain-specific age effects (Güsten et al., 2021). Using cross-validation we show better out-of-sample prediction for the GCMR as compared to Rasch and 2HT model. Finally, an adaptive test using the GCMR is simulated, showing that the test length necessary to obtain reliable ability estimates can be significantly reduced compared to a non-adaptive procedure. The GCMR allows to model trial-by-trial performance and to increase the efficiency and reliability of recognition memory assessments.
\end{abstract}




\section{Introduction}

Research on human memory has long seen a quest for adequate cognitive models to describe the relevant processes underlying behavioural responses. Modeling of recognition memory has largely seen two classes of stochastic models: signaldetection theory (SDT) models (Macmillan and Creelman, 2004) and threshold models (Snodgrass and Corwin, 1988). Both make predictions about the latent processes leading to mnemonic decision making. SDT posits distributions over the underlying memory strength which partly overlap. Thus the respondent is never in a pure state of stimulus recognition or rejection, but rather makes a qualified decision depending on a hypothesized response criterion. Threshold models, on the other hand, assume that latent memory representations are categorical and therefore disjoint. A stimulus is either remembered or not, depending on a subject-specific threshold. When the threshold is not reached, a subject is not able to recognize or detect a stimulus, but is still able to answer correctly as a result of pure guessing. In the simple case of old-new recognition memory tasks, the standard SDT model uses an ability discrimination parameter $d^{\prime}$ (d-prime) with the corresponding response bias c, and assumes equal variances of the underlying noise and signal distributions. A popular threshold model is the 2 High-Threshold (2HT) Model, which belongs to a broader class of so-called multinomial processing tree (MPT) models, whose latent processes can be visualized as paths in a decision tree. In recognition memory, where performance is typically estimated based on a single false alarm rate and a hit rate, a common threshold for recognition and rejection is often assumed in order to be identifiable (Snodgrass and Corwin, 1988). The corresponding threshold parameter is then called $\mathrm{Pr}$, and given that it can be estimated by substracting the false alarm rate from the hit rate, it is often simply called "corrected hit rate". The $2 \mathrm{HT}$ model also contains a guessing parameter called $\mathrm{Br}$. In the following, we will use the terms SDT and 2HT to denote the standard SDT model and the $2 \mathrm{HT}$ model with a common threshold, respectively.

Both the SDT and the 2HT model distinguish between memory sensitivity and response bias or guessing. Importantly, it has been shown for both models that sensitivity and response bias are theoretically independent, and thus can be separately manipulated in behavioural experiments (Snodgrass and Corwin, 1988). Response biases might differ across populations and may be of relevance in clinical diagnostics of various neurological disorders. For instance, a more liberal response bias has been observed in old age (Huh et al., 2006). Moreover, Snodgrass and Corwin (1988) found that while both amnesia and dementia (Huntington's [HD], Parkinson's Dementia [PD] and Alzheimer's Dementia $[\mathrm{AD}])$ patients show weaker memory compared to cognitively normal subjects, only dementia patients exhibit a more liberal response bias. AD patients' response bias was found to be similar across different recognition tasks, while in healthy older adults it varied with task and stimulus type (Beth et al., 2009). When comparing models, Snodgrass and Corwin (1988) noted that ability and bias parameters from the $2 \mathrm{HT}$ model were generally more sensitive to disease 
status than $d$ '. More specifically, the authors observed differential forgetting as a function of disease status for $\operatorname{Pr}$ but not $d$ '. That is, diagnostic groups differed with respect to response bias only when measuring $B r$, but not $c$. What is more, only $\mathrm{Br}$ became more liberal over trials for $\mathrm{PD}$ and $\mathrm{AD}$ patients as compared to healthy subjects. These findings point to the $2 \mathrm{HT}$ model as a sensitive and promising model for measuring disease-related cognitive impairment and memory decline.

\section{IRT and computerized adaptive testing}

Item response theory (IRT) is a psychometric framework which provides sophisticated tools to define, estimate and analyze individual differences in terms of latent (or noise-free) characteristics of participants. A typical example of such a latent psychological variable (or construct) is the ability of a participant to discriminate between items in a recognition memory task. Other examples are verbal and quantitative reasoning, which are assessed in the Graduate Record Examination (GRE) and represent an admission criterion to many graduate schools in the US and Canada. As a measurement framework, IRT provides several advantages compared to the classical test theory (CTT), upon which most conventional cognitive modeling relies (Brown et al. 2017). Given independence of item and person-specific parameters, IRT estimates of latent constructs do not depend on a given test or item. Using IRT, measurement error can be assessed at the latent construct level, which is typically not constant across the latent dimension (Thomas, 2019). For instance, a test that mostly employs items of average difficulty will be more precise for subjects of average ability, as compared to very high or low ability. Indeed, the fact that measurement error depends on the participant's level of ability, has been observed for measures of cognition (Gavett and Horwitz, 2011; Pedraza et al., 2011), personality (Ranger and Ortner, 2011; Spence et al., 2012), and psychiatric symptoms (Olino et al. | 2012). IRT provides tools to model this error directly and may therefore improve clinical assessments.

One promising use of IRT in modern clinical assessment is its implementation in Computerized Adaptive Testing (CAT) (Walter et al., 2007; Glas and van der Linden, 2003). In CAT, test items are adaptively administered to participants in an optimal fashion so as to maximize the information gain and thereby the reliability of assessments. As a consequence, using CAT can lead to much shorter test lengths and higher efficiency. Moreover, as the standard error of the estimate can be obtained on a trial-by-trial basis, it can flexibly be used as a criterion set by the experimenter in order to reach a desired level of certainty. These are some of the beneficial properties that have led to widespread use of CAT in educational (e.g. TOEFL, GRE) as well as cognitive assessment. One prominent example is the NIH Toolbox (Weintraub et al., 2013), which contains two adaptive language tests: the NIH-TB Oral Reading Recognition Test and the NIH-TB Picture Vocabulary Test (Gershon et al., 2014).

Finally, CAT has also gained increasing popularity in clinical assessment. For instance, it has been used to assess depression (Gibbons et al. 2012), anx- 
iety (Gibbons et al., 2014) as well as personality disorders (Gibbons et al. 2020). Other studies in the domain have shown its usefulness via simulated tests (Reise and Henson, 2000, Sunderland et al., 2017). Nonetheless, despite these promising examples and benefits that CAT provides, its overall use in clinical assessment is still relatively scarce (Thomas, 2019).

\section{Combining cognitive models with IRT}

By accounting for item and person heterogeneity and providing more sophisticated tools to account for measurement error, IRT could benefit the study of recognition memory. However, IRT was not conceived to provide psychologically plausible explanations of decision-making processes in old-new recognition tasks as is the case in the cognitive models introduced above (Thomas et al., 2018 , DeCarlo, 2019). For instance, IRT models do not traditionally model response bias or guessing as a person parameter, as do the $2 \mathrm{HT}$ or SDT models. In recent years, several authors have argued for an approach that combines mathematical cognitive models with psychometric IRT models (Karabatsos and Batchelder, 2003, Batchelder, 2010; Brown et al., 2017) and first combined approaches with SDT have been proposed regarding recognition memory models in particular (Thomas et al. 2018, DeCarlo, 2019). Others have introduced models that merge IRT with MPT, where transition probabilities in the MPT models are the result of an IRT model process (Batchelder, 2010, Boeck and Partchev, 2012 Jeon and Boeck, 2015). For instance, Boeck and Partchev (2012) presented the IRTTree framework, that combines MPT with IRT models represented as Generalized Linear Mixed Effects Models. Other authors have proposed to describe MPT parameters by the Rasch model from IRT (Batchelder, 2010; Karabatsos and Batchelder, 2003). Specifically, they applied the Rasch model to an extension of the 2HT model, the General Condorcet Model (GCM). Despite the two models' formal similarity, the $2 \mathrm{HT}$ is mainly used to separate latent ability from response bias, while the main purpose of the GCM is to study cultural consensus regarding the correctness of responses (Batchelder and Romney, 1988). Hence, while in the $2 \mathrm{HT}$ model - as is typical in recognition memory - the expected answer is fixed by design, it is the parameter of interest in the GCM.

Here we propose a new model for old-new recognition memory further denoted as GCMR (General Condorcet Model for Recognition Memory). The GCMR combines the Rasch model from IRT with the 2HT model. As mentioned above, the $2 \mathrm{HT}$ is a widely used model in recognition memory, which has been suggested to possess higher sensitivity in clinical assessment when compared to $d^{\prime}$ 'Snodgrass and Corwin (1988). In what follows we introduce the GCMR more formally and subsequently conduct three studies to validate the approach. In a simulation study, we explore potential advantages of the approach in two typical recognition memory task scenarios, testing under which conditions ability estimates for GCMR diverge from its constituting models, the Rasch and the 2HT model, individually. We further validate the model using a real dataset consisting of large recognition memory sample collected online via Amazon Mechanical Turk. Finally, we investigate the GCMR's potential for 
CAT, such as benefits for test efficiency, time savings and valuable estimates of conditional measurement error.

\section{Methods}

In what follows we briefly review the technicalities of the behavioral models from which we derive the GCMR and close this section with a presentation of our validation strategy using synthetic and real datasets.

\subsection{Behavioral Models}

We begin by describing the $2 \mathrm{HT}$ and the Rasch model more formally. The GCMR is then introduced, which integrates the two models and posits that the memory process is a function of both person ability and item difficulty, while also accounting for response biases.

\section{HT model}

In the context of recognition memory experiments, participants typically observe a series of (visual or auditory) stimuli. After each stimulus (or experimental trial), they are asked to indicate whether it was old or new. We further denote the participant's response of a trial using random variable $U$ taking value "old" or "new". We further use variable $t$ to indicate trial types taking on the values $t=$ target and $t=$ foil. Then the $2 \mathrm{HT}$ (or "corrected-hits") model specifies "hit" (correct recognition) and "false alarm" (false recognition) in terms of event probabilities as follows:

$$
\begin{gathered}
P(\text { hit })=P(U=\text { old } \mid t=\text { target })=P r+(1-P r) \gamma \\
P(\text { false alarm })=P(U=\text { old } \mid t=\text { foil })=(1-P r) \gamma,
\end{gathered}
$$

where $\operatorname{Pr}$ represents the discrimination parameter, and refers to the probability of "knowing" the correct answer, given that a target or foil was presented. The guessing parameter $\gamma$ denotes the probability of responding "old" when the correct answer is unknown, reflecting a guessing tendency or response bias. We can easily see that $\operatorname{Pr}$ is the difference of the hit rate and false alarm rate.

The proposed GCMR merges the $2 \mathrm{HT}$ and the Rasch model. As is common in IRT, the correctness of a response is modeled. For consistency, we reformulate the above $2 \mathrm{HT}$ model using variable $Y=1$ to code a correct response and $Y=0$ for an incorrect response

$$
p(Y=1 \mid \operatorname{Pr}, \gamma, t)=\operatorname{Pr}+(1-r) \gamma^{t}(1-\gamma)^{(1-t)}
$$

in a trial with trial type $t=1$ for target trials and $t=0$ for foil trials. For the remainder, we will refer to $\operatorname{Pr}$ as $\theta_{2 H T}$, where $\theta$ denotes an ability parameter. 


\section{Rasch model}

Next we briefly describe the Rasch model (Rasch, 1980). Here, the probability of a correct response is assumed to be a function of both person ability and item difficulty. The model uses the logit function to map the real-valued ability and difficulty parameters to probabilities. The probability of answering correctly is given by the logit of their difference:

$$
p(Y=1 \mid \theta, \beta)=\frac{e^{(\theta-\beta)}}{1+e^{(\theta-\beta)}},
$$

where $\theta$ and $\beta$ refer to the person ability and item difficulty, respectively. The key assumption is that only the difference of both parameter affects probability of a correct response. The function approaches 1 when ability strongly exceeds item difficulty and 0 if the item is much too difficult for a certain person performing the task, e.g. on recognition memory. It is worth noting that neither trial type nor for response bias is accounted for.

\section{GCMR - combining Rasch and 2HT model}

The 2HT model assumes equal item difficulties, which is rarely the case in recognition memory tasks. The GCMR's main purpose is to account for item heterogeneity. In order to do so, we can model the discrimination stage of the $2 \mathrm{HT}$ as depending on a latent person ability $\theta$ and an item difficulty $\beta$ given by the Rasch model (eq. 4), obtaining a model that accounts for person ability, item difficulty and response bias:

$$
p(Y=1 \mid \theta, \beta, \gamma, t)=\frac{e^{(\theta-\beta)}}{1+e^{(\theta-\beta)}}+\left(1-\frac{e^{(\theta-\beta)}}{1+e^{(\theta-\beta)}}\right) \gamma^{t}(1-\gamma)^{(1-t)}
$$

The depicted model is equivalent to the Rasch extension of the GCM, with one important difference: unlike for the GCM, trial type $t$ is not a parameter, but an experimental design variable. In other words, the expected response for each trial is set by the experimenter instead of having to be estimated. This is a common feature of recognition memory tasks, whereas GCM was conceived to infer the expected response as a function of cultural consensus in the domain of sociology and cross-cultural studies (Oravecz et al., 2013; Batchelder and Romney, 1988). 


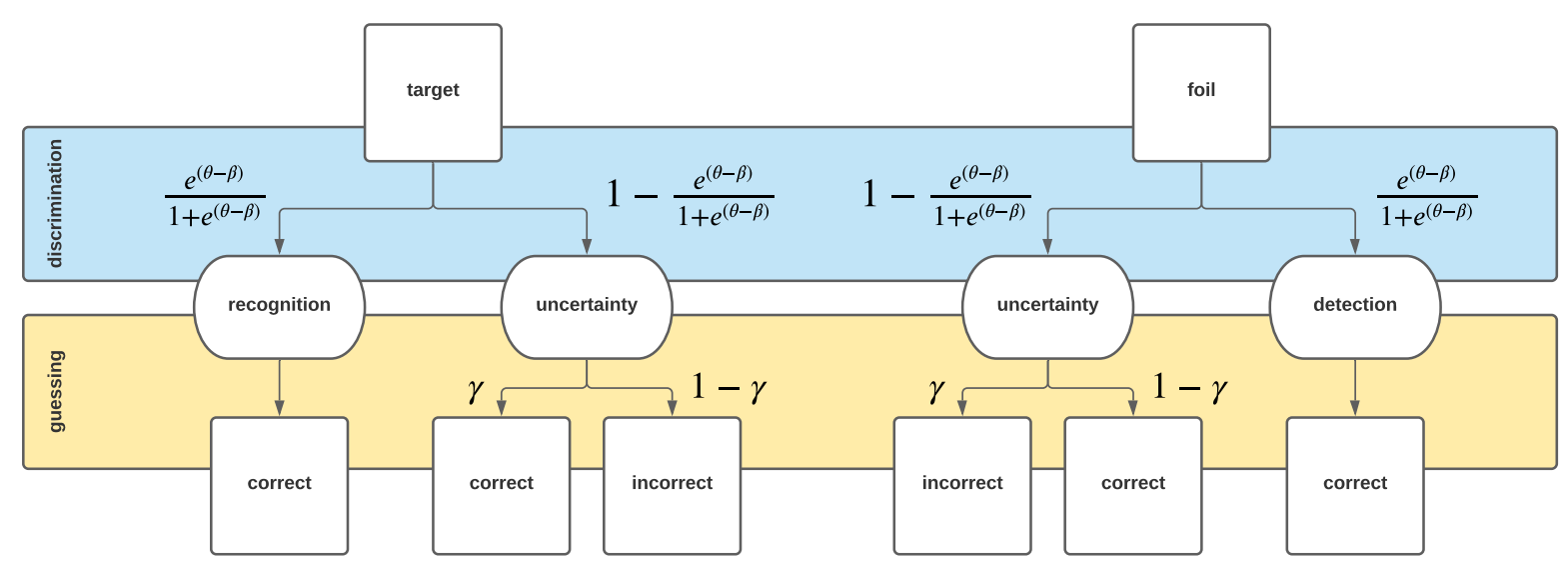

Figure 1: Schematic tree diagram of the GCMR

The proposed model can be interpreted using two decision trees, one for each trial type. The trees are divided into two latent processes: discrimination and guessing. Transition probabilities follow a Rasch model in the discrimination process, and are defined by parameter $\gamma$ in the guessing process.

\subsection{Model inference}

Here we follow the Bayesian paradigm for estimation and model inference (Gelman et al., 2013), given that it has been successfully applied to models from the GCM family Karabatsos and Batchelder (2003). In addition, a Bayesian treatment allows for flexible incorporation of prior knowledge from previous studies recognition memory, which may be available once the model has been tested in different domains or samples. Moreover, Bayesian modeling provides an elegant way to analyse both parameter and model uncertainty, a very attractive feature in the context of clinical assessment. Here, the parameters for all models were estimated using Markov-Chain Monte-Carlo (MCMC) sampling, implemented in Stan via the R interface Stan Development Team (2020). The use of MCMC sampling methods is omnipresent in the estimation of Bayesian models, as the integrals from the marginal distribution are typically intractable and cannot be estimated using conventional analytical methods (Karabatsos and Batchelder, 2003).

\subsection{Simulation study}

We performed two validation studies to test face validity of the GCMR in the context of synthetic data, real data as well as its potential for adaptive testing. The goal of the simulation study was to assess the GCMR in scenarios of potential real studies, varying key parameters. We investigated under what conditions its ability estimates would align or differ from both the Rasch and $2 \mathrm{HT}$ 
model. As per construction our prior expectation was that the GCMR might capture information that the two single models do not provide. Furthermore, the manipulations tested here might help inform experimenters whether the use of GCMR is warranted for their specific data and problem at hand.

Concretely, we simulated response data of 100 subjects performing a task with 50 items ( 25 foils, 25 targets). A total of 20 simulations were run for each of the parameter combinations. We then estimated how well 2HT, Rasch and GCMR were able to recapture ground truth individual differences under different manipulations, including high bias, difficulty and missingness. For each model, we compared GCMR ground truth with estimated parameters using the Pearson correlation coefficient, since the model parameters live on different scales and hence a scale-free metric was warranted.

\section{Default parameter values}

Ability $\theta$ was modeled with a standard normal distribution, as is common in IRT, that is $\theta \sim N(0,1)$. The bias parameter $\gamma$ the GCMR is introduced via the $2 \mathrm{HT}$ model and the GCM, and there it is usually modeled as a probability on the unit scale, using the beta distribution as a prime candidate (Oravecz et al. 2013 Karabatsos and Batchelder, 2003). Here, we follow this parametrization. Given that the beta distribution has no explicit mean parameter, its mean $\mu_{\gamma}$ was varied indirectly by manipulating the distribution's shape parameters $\alpha_{\gamma}$ and $\beta_{\gamma}$. In so doing, we varied its mean while keeping the variance constant. The three levels of bias mean $\mu_{\gamma}$ were modeled as follows:

\begin{tabular}{r|r}
\hline$\mu_{\gamma}$ & \\
\hline 0.5 & $\operatorname{Beta}(10,10)$ \\
0.75 & $\operatorname{Beta}(11.0625,3.6875)$ \\
0.9 & $\operatorname{Beta}(5.904,0.656)$ \\
\hline
\end{tabular}

\section{Varying test difficulty and response bias}

The first manipulation aimed to investigate the potential key advantage of GCMR compared to the Rasch and $2 \mathrm{HT}$ model, namely accounting for item difficulty in addition to trial type and response bias. We were interested in how parameter estimation is affected when overall performance approaches chance level. Low performance is likely to be encountered in clinical populations as well as ageing cohorts, when these are compared to healthy young subjects on the same test. Furthermore, diverging patterns of response bias have been observed in these cohorts. Specific failure to correctly estimate ability under low performance or diverging response bias is therefore of paramount importance to clinicians. Here we varied average test difficulty as a means to manipulate performance, and explored how the effect of difficulty would interact with varying levels of response bias. The true parameter values of average item difficulty $\mu_{\beta}$ and average response bias $\mu_{\gamma}$ were varied as follows: 


\begin{tabular}{rr}
\hline$\mu_{\beta}$ & $\mu_{\gamma}$ \\
\hline-2 & 0.5 \\
0 & 0.75 \\
2 & 0.9 \\
\hline
\end{tabular}

Parameter levels were crossed, leading to a grid of 9 parameter combinations.

\section{Varying missingness and response bias}

One fundamental limitation of the $2 \mathrm{HT}$ model is that person ability and itemor test difficulty are not necessarily independent. Thus $\theta_{2 H T}$ does not define a test-independent latent construct (dimension) as is aimed for in IRT (Thomas, 2010). As a consequence, $2 \mathrm{HT}$ model estimates are only comparable when using the same or a truly parallel test. Having to compare participants performance across different tests is a situation well familiar to many experimenters. This can arise when participants do not respond to all items, or in the context of longitudinal assessment. In this part of the simulation study we manipulated the amount of missing data, effectively creating varying subtests. Here, we tested a simple kind of missingness, where a fixed number of trials per participant were randomly deleted. Note that this should not change the mean test difficulty, but its variance. We expected that this would create more noise in $\theta_{2 H T}$ estimates given its dependence on test difficult, as compared to ability estimates from the GCMR and possibly the Rasch model, which both directly account for item difficulty. To test this, the percent of missing data per subject $\mathrm{p}$ (missing) and the true parameter values of $\mu_{\gamma}$ were varied as follows:

\begin{tabular}{rr}
\hline $\mathrm{p}$ (missing) & $\mu_{\gamma}$ \\
\hline 0 & 0.5 \\
0.25 & 0.75 \\
0.5 & 0.9 \\
\hline
\end{tabular}

For model estimation, we used the priors as specified in Appendix A.

\subsection{Real data study I: Object-scene memory task in a web- based lifespan sample}

In order to validate the GCMR on real data, we applied it to a large sample dataset (Güsten et al., 2021) in which participants performed a recognition memory task (Berron et al. | 2018). In this particular study, participants also performed the task on differing subsets of a large item pool. The use of (pseudo)random subsets of a larger stimulus pool is a common strategy in recognition memory research to minimize learning and stimulus-repetition effects (Morcom et al. 2003, Kantner and Lindsay, 2012). Thus, this study represents a common application scenario characterized by high missingness (or sparsity). As 
mentioned earlier, for conventional estimates such as $\theta_{2 H T}$ to be meaningful, equal test difficulty must be assured. The data set used here very likely violates the assumption of equal difficulty of all the subtests. GCMR on the other hand does not rely on tests being equally difficult, as person and item parameters are estimated independently. Moreover, by modeling item heterogeneity, the GCMR is able to capture trial-by-trial variance, which cannot be predicted by the $2 \mathrm{HT}$ model.

Here, we first compared model evidence using Bayesian model stacking, predicting that GCMR would show better (out-of-sample) predictive performance than both the $2 \mathrm{HT}$ and Rasch model. We then explored face validity of GCMR, investigating whether the pattern of results previously found with conventional 2HT modeling (Güsten et al., 2021). In particular, the study found that task performance decreases with age, and that the age-related decrease depends on the stimulus domain, being stronger for stimuli representing objects than for scene stimuli. In addition, we explored whether the $2 \mathrm{HT}$ model and the GCMR show similar effects of response bias as a function of both age and the age by domain interaction.

\section{Sample and stimulus material}

We used a large web-based observational study of 1554 adults (18-77 years, $\mathrm{M}=37.19, \mathrm{SD}=11.61,61 \%$ females) that was analyzed by (Güsten et al. 2021). Participants had on average only responded to $2.42 \%$ of stimuli of a large item-pool, resulting in a highly sparse response matrix (indicating high missingness). They were tested on previously fixed subsets of the entire item pool, which themselves were subject to further randomizations of the stimulus versions presented. Therefore, missingness was induced by design, given that the following quantities varied across participants: (1) the item overlap between subsets, (2) the number of items tested. All in all, this resulted in a rather unbalanced study design. Consequently, a larger sample increases numbers of available responses for each stimulus to eventually result in more reliable item parameters.

\section{Task}

Participants performed a 2-back mnemonic discrimination task, in which they had to discriminate between target and lure versions of object and scene stimuli. It constitutes a version of the object-scene task (Berron et al., 2018) that we had previously employed. The task consisted of several trials containing two item presentations followed by two identical (target) or very similar different (lure) test items each. Subjects had to respond to each test stimulus with old/new judgments pressing the left or right arrow key, whereby each trial either contained only objects or scenes. 


\section{Model comparison}

To assess model fit, we used efficient approximate leave-one-out (LOO) crossvalidation for Bayesian models using Pareto smoothed importance sampling (PSIS) (Vehtari et al., 2019). The aim of cross-validation is to test out-ofsample prediction and penalize model complexity. We compared the three models (GCMR, Rasch, 2HT) using Bayesian stacking, which maximizes the leave-one-out predictive density of the combination distribution, finding the optimal linear combining weights that maximize the leave-one-out log score (Yao et al. 2017). This model comparison approach identifies the best model as the one obtaining the highest stacking weight.

\section{Population analysis: linear mixed-effects model}

Posterior means of ability parameters from the GCMR, Rasch and 2HT model were separately estimated for task data from each domain (objects and scenes). Estimates were then pooled, scaled and centered for each model and subsequently entered into a group analysis. Here we used linear mixed effects modeling with the lme4 package in $\mathrm{R}$ (Bates et al., 2015), in order to assess effects of age, stimulus domain and the age $\mathrm{x}$ stimulus domain interaction. Random intercepts were included for each participant. This enables accounting for the nested nature of the study design, including repeated measures for each subject. Furthermore, relevant design variables were entered as covariates into the model.

\subsection{Real data study II: CAT}

In this last experiment, we explored the potential of the GCMR to be used in CAT. CAT requires item difficulty to be modeled and adaptively matched with a participant's ability in a trial-by-trial fashion. This requires modeling of item parameters and cannot be performed using conventional detection models such as the $2 \mathrm{HT}$ model. Notice that unlike the Rasch model, GCMR possesses two person-specific parameters (i.e. ability and bias) which are fitted simultaneously, and can therefore be seen as a multidimensional IRT model. Here, we tested in a second application study whether (A) this parameter inference can be reliably performed; and whether (B) CAT can accelerate parameter convergence compared to conventional fixed tests. For one thing, this may significantly improve test efficiency for clinical applications.

\section{Sample}

We focused on the same web-based sample as in the above real data study. In a first step, 200 participants (denoted as the CAT sample) were sampled at random for inclusion in the adaptive test experiment. Data from the remaining 1354 participants (calibration sample) was used to calibrate the item sets. 


\section{Item calibration}

As commonly done in CAT, we restricted the test to a subset of items that showed good model-data fit. Item parameters were fitted using the calibration sample and estimated via MCMC sampling in Stan, and posterior mean values subsequently extracted as point estimates of the parameters. Given that a sufficient amount of trial data per participants was needed for the CAT algorithm, a single model was fitted on the combined object and scene data. For item selection, we used the common infit statistic (Smith et al., 2008), for which scores around 1 or lower indicate a good model-data fit. Underfitting items were excluded based on an infit threshold of 1.3 (Smith et al., 2008)

\section{CAT procedure and algorithm}

Response data from each subject of the CAT sample was used to simulate an adaptive test, mainly following the strategy by Reise and Henson (2000). All participants were given a starting latent ability of $\theta=0$. Then, the item with the highest information given that trait level was administered (see item information function in Appendix B). The algorithm, which was based on the CatR toolbox (Magis and Raîche, 2012) and modified to incorporate the GCMR, used Maximum Fischer Information (MFI) as the item choice criterion. Given the participant's response, a new ability was assessed using maximum-a-posteriori (MAP) estimates (Samejima, 1969), with ability priors being standard normally distributed. The above procedure of computing the item information at the participant's current estimated ability, presenting the most informative item, collecting the response and reestimating ability was repeated for 80 trials. The CAT was restricted to this length so as to remain comparable to common paradigms in the field. Also, further extending the length would have prevented the algorithm to choose adaptively fitting items, given that item presentations per participants were set by the existing data $(\mathrm{M}=130.67, \mathrm{SD}=3.23)$.

\section{Convergence analysis}

In order to evaluate the efficiency of the approach, we calculated the following two statistics after every CAT trial (Reise and Henson, 2000): (1) the correlation of individual trial-wise updated parameter estimates with the corresponding full-sample estimates and (2) trial-wise standard errors of ability estimates $(\theta)$. Notice that CAT estimates were always based on up to 80 trials, while the full estimates were based on the entirety of trials seen by the participant in the original data.As noted earlier, CAT cannot be performed with conventional recognition models such as the $2 \mathrm{HT}$. In order to show the benefit of using CAT, we calculated trial-wise ability estimates of GCMR and 2HT using the original, non-adaptive item administration order for comparison. We then assessed to what extent the increase of trial-wise GCMR ability correlation with full estimates can be accelerated by an adaptive administration order. 


\section{Results}

\subsection{Simulation study}

\section{Varying test difficulty and response bias}

Over all 9 combinations of test difficulty and response bias, the highest mean correlation with ground truth and lowest standard error (SE) of ability estimates was observed for the GCMR $(\rho=.885, \mathrm{SE}=.0065)$, followed by Rasch $(\rho=.857, \mathrm{SE}=.0083)$ and $2 \mathrm{HT}(\rho=.853, \mathrm{SE}=.0086)$ estimates, as summarized in Figure 2A. All models exhibited high correlations with ground truth when test difficulty matched the average participant ability (GCMR: $\rho=.912, \mathrm{SE}=.0048$; Rasch: $\rho=.891, \mathrm{SE}=.0069$; $\operatorname{Pr}: \rho=.893, \mathrm{SE}=.0070)$ and a low correlation when test difficulty was higher than average ability. The GCMR $(\rho=.852, \mathrm{SE}=.0088)$ showed better recovery than the Rasch $(\rho=.794, \mathrm{SE}=.0126)$, and 2HT $(\rho=.796$, $\mathrm{SE}=.0125$ ) model. Regarding effects of response bias, all three explored models performed worst when bias was balanced $(\bar{\gamma}=.5$, GCMR: $\rho=.874, \mathrm{SE}=.0070$, Rasch: $\rho=.836, \mathrm{SE}=.0086,2 \mathrm{HT}: \rho=.827, \mathrm{SE}=.0095)$ and best when bias was more pronounced $(\bar{\gamma}=0.9$, GCMR: $\rho=.904, \mathrm{SE}=.0053$, Rasch: $\rho=.886, \mathrm{SE}=.0065$, $2 \mathrm{HT}: \rho=.886, \mathrm{SE}=.0062)$. When test difficulty was low $(\bar{\beta}=-2)$, the $2 \mathrm{HT}$ model was found to be more affected by lower average response bias (0.9 to 0.5) than the GCMR or the Rasch model (GCMR: $\Delta \rho=-.026$, Rasch: $\Delta \rho=-.029,2 \mathrm{HT}$ : $\Delta \rho=-.053)$. Under high test difficulty $(\beta=2)$, both Rasch and the $2 \mathrm{HT}$ model were more negatively affected than GCMR (GCMR: $\Delta \rho=-.041$, Rasch: $\Delta \rho=$ $-.080,2 \mathrm{HT}: \Delta \rho=-.083)$.

\section{Varying missingness and response bias}

The proposed GCMR $(\rho=.876, \mathrm{SE}=.0074)$ showed a higher mean correlation and lower SE than the Rasch $(\rho=.854, \mathrm{SE}=.0083)$ and $2 \mathrm{HT}$ model $(\rho=.885$, $\mathrm{SE}=.0065$ ). Again, the GCMR performed best across all explored parameter combinations in recovering its ground truth (Figure 2B). All models showed higher correlations when missingness was low $(0 \%$ missing, GCMR: $\rho=.913$, $\mathrm{SE}=.0044$, Rasch: $\rho=.894, \mathrm{SE}=.0055,2 \mathrm{HT}: \rho=.895, \mathrm{SE}=.0054)$, and performed worst when missingness was high $(50 \%, \mathrm{GCMR}: \rho=.826, \mathrm{SE}=.0128$, Rasch: $\rho=.802, \mathrm{SE}=.0135,2 \mathrm{HT}: \rho=.784, \mathrm{SE}=.0054)$. It is worth mentioning that while the Rasch and the $2 \mathrm{HT}$ model performed comparably under low and medium missingness, the $2 \mathrm{HT}$ performed worse than the Rasch model under high missingness. As above, all models performed worst when response bias was balanced $(\bar{\gamma}=.5, \mathrm{GCMR}: \rho=.874, \mathrm{SE}=.0070$, Rasch: $\rho=.836, \mathrm{SE}=.0086,2 \mathrm{HT}: \rho=.827$, $\mathrm{SE}=.0095)$ and best when bias was more pronounced $(\bar{\gamma}=0.9$, GCMR: $\rho=.904$, $\mathrm{SE}=.0053$, Rasch: $\rho=.886, \mathrm{SE}=.0065,2 \mathrm{HT}: \rho=.886$, $\mathrm{SE}=.0062)$. When missingness was high, the $2 \mathrm{HT}$ and Rasch model were found to be more affected by a decrease in bias than the GCMR $(\gamma=0.9$ to 0.5 , GCMR: $\Delta \rho=-.084$, Rasch: $\Delta \rho=-.102$, 2HT: $\Delta \rho=-.105)$, whereas SE increased similarly for all three models (GCMR: $\Delta \mathrm{SE}=.0144$, Rasch: $\Delta \mathrm{SE}=.0130,2 \mathrm{HT}: \Delta \mathrm{SE}=.0135$ ). 

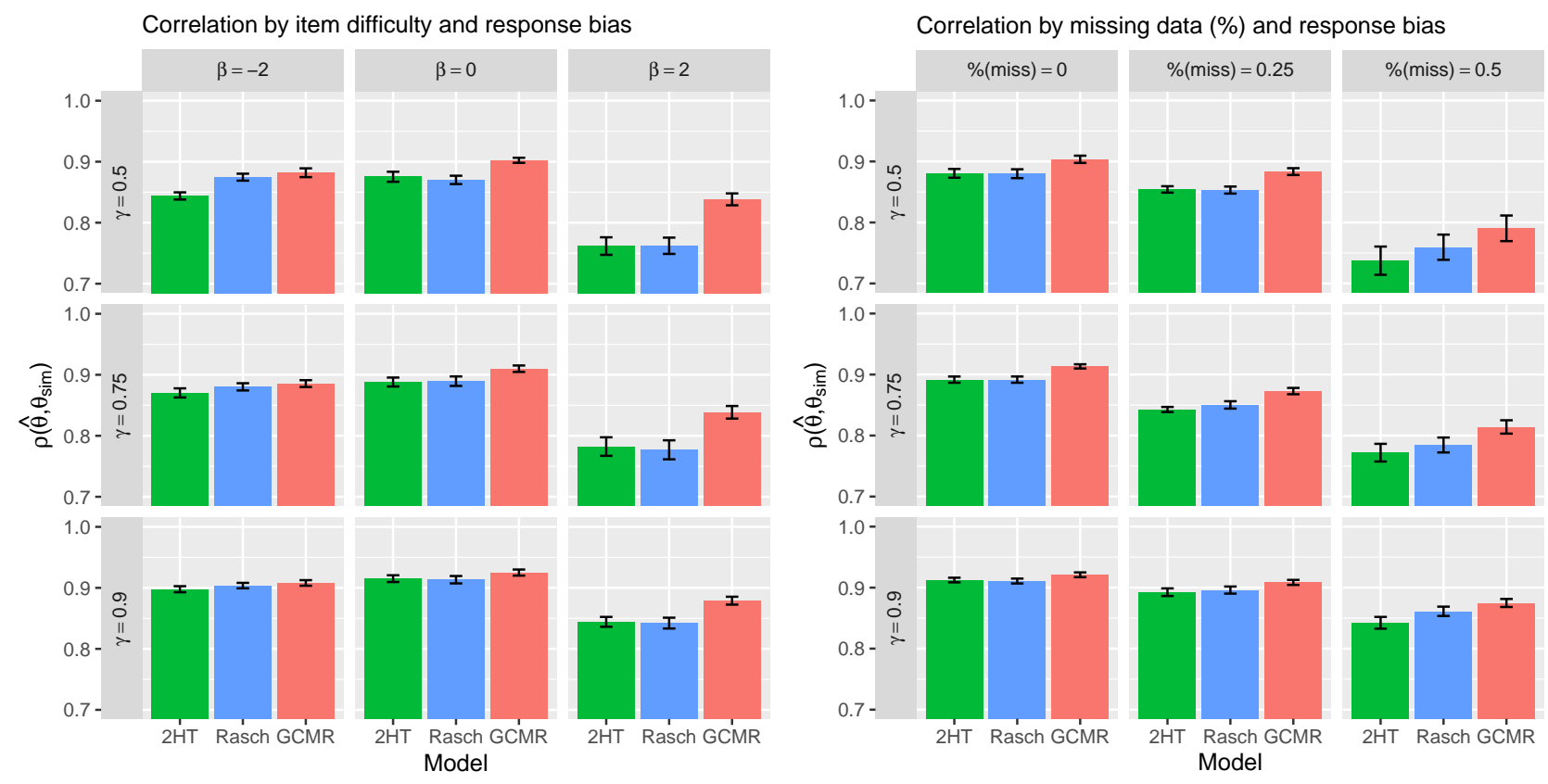

Figure 2: Correlation of estimated and true scores as a function of response bias and item difficulty (A) or missing responses (B)

A Ground truth correlation for all models is highest when difficulty matches average ability, while it decreases for Rasch and 2HT estimates under high difficulty, especially when response bias is low. In addition, $2 \mathrm{HT}$ correlation with ground truth decreases under low difficulty and low bias. B While all ability estimates correlate strongly with ground truth when responses are complete, GCMR estimates show good recovery under high degree of missing responses, while ground truth correlation for Rasch and 2HT estimates decreases. 


\subsection{Real data study I: object-scene memory task in a web- based lifespan sample}

\section{Model comparison: LOO-CV}

All models seemed to converge well (Appendix C) as indicated by low $\hat{R}$ statistics (Gelman and Rubin, 1992). As for the LOO-crossvalidation, Bayesian stacking resulted in the GCMR obtaining almost all the mixing weight, both in the object condition $\left(w_{G C M R}=0.999, w_{2 H T}=0.000, w_{R a s c h}=0.001\right)$, as well as the scene condition $\left(w_{G C M R}=0.980, w_{2 H T}=0.020, w_{R a s c h}=0.000\right)$. This suggests that the GCMR has higher (out-of-sample) predictive power and model evidence than both Rasch and $2 \mathrm{HT}$ for trial-level data from this object-scene memory task.

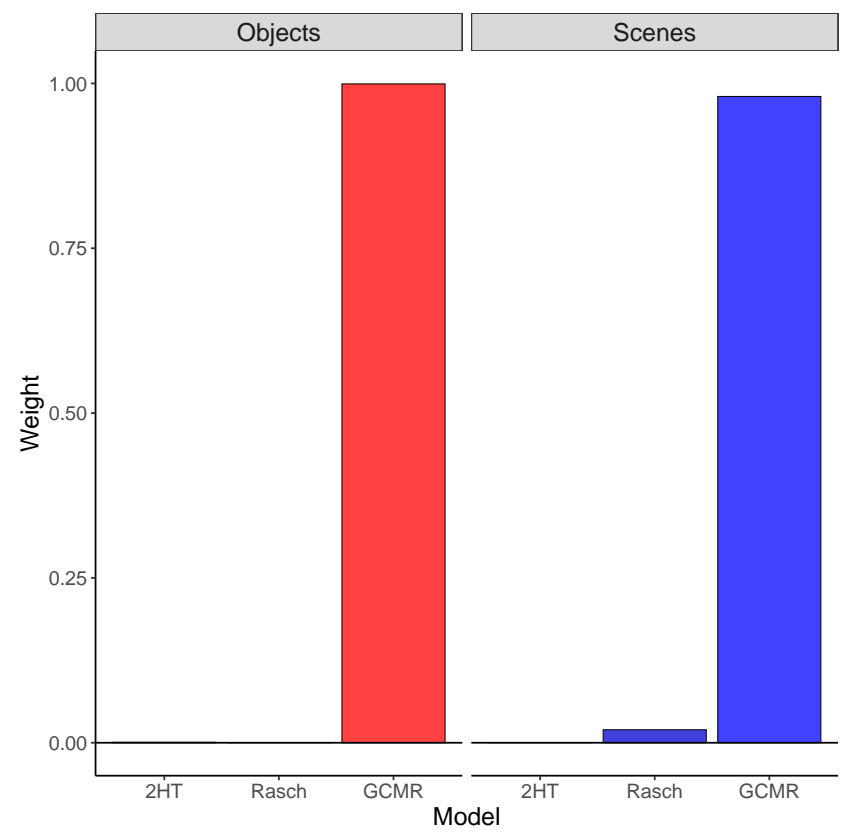

Figure 3: Leave-one-out cross-validation (LOO-CV) model weights obtained from Bayesian stacking. For both domains, GCMR has highest stacking weight, suggesting best predictive performance for trial-based data in the object-scene task.

\section{Population analysis: linear mixed-effects model}

In this study, we observed a negative main effect of age on ability $\theta$ for all three models (GCMR: $F=12.90, \mathrm{p}=0.0003$, Rasch: $F=15.79, \mathrm{p}=0.0001,2 \mathrm{HT}$ : $F=21.22, \mathrm{p}<0.0001$ ), as well as a robust age by domain interaction for both the GCMR and $2 \mathrm{HT}$ model, driven by steeper decline in object performance 
across age. This replicates our previous work in suggesting that mnemonic discrimination of highly similar stimuli decreases with age, with an additional domain-dependency Güsten et al. (2021). For the Rasch model, on the other hand, the interaction effect was not as robust (GCMR: $F=7.13, \mathrm{p}=0.0077$, Rasch: $F=3.25, \mathrm{p}=0.0716,2 \mathrm{HT}: F=5.65, \mathrm{p}=0.0176)$.

As for response bias, we observed an age-related increase for both the GCMR $(F=6.45, \mathrm{p}=0.0112)$ and the $2 \mathrm{HT}(F=6.57, \mathrm{p}=0.0105)$ model, yielding additional evidence for a more liberal response bias in older participants. Moreover, we found stronger bias increase for the stimulus domain of objects compared to scenes in the $2 \mathrm{HT}$ model $(F=5.20, \mathrm{p}=0.0228)$, but not the $\operatorname{GCMR}(F=$ $2.36, \mathrm{p}=0.1244)$.

Table 1: Anova Table $\theta$

\begin{tabular}{lrrrrrr}
\hline GCMR & Sum Sq & Mean Sq & NumDF & DenDF & F value & Pr $(>$ F $)$ \\
\hline Age & 7.27 & 7.27 & 1.00 & 1522.00 & 14.72 & 0.0001 \\
Domain & 3.20 & 3.20 & 1.00 & 1552.00 & 6.49 & 0.0110 \\
Item Set & 16.57 & 0.57 & 29.00 & 1522.00 & 1.16 & 0.2577 \\
Version Order & 2.65 & 2.65 & 1.00 & 1522.00 & 5.37 & 0.0207 \\
Age:Domain & 3.52 & 3.52 & 1.00 & 1552.00 & 7.13 & 0.0077 \\
\hline Rasch & & & & & \\
\hline Age & 6.47 & 6.47 & 1.00 & 1522.00 & 11.77 & 0.0006 \\
Domain & 1.75 & 1.75 & 1.00 & 1552.00 & 3.18 & 0.0748 \\
Item Set & 19.87 & 0.69 & 29.00 & 1522.00 & 1.25 & 0.1722 \\
Version Order & 2.01 & 2.01 & 1.00 & 1522.00 & 3.66 & 0.0560 \\
Age:Domain & 1.79 & 1.79 & 1.00 & 1552.00 & 3.25 & 0.0716 \\
\hline 2HT & \multicolumn{7}{c}{} & & & \\
\hline Age & 10.44 & 10.44 & 1.00 & 1522.00 & 22.12 & 0.0000 \\
Domain & 79.05 & 79.05 & 1.00 & 1552.00 & 167.56 & 0.0000 \\
Item Set & 28.47 & 0.98 & 29.00 & 1522.00 & 2.08 & 0.0007 \\
Version Order & 28.24 & 28.24 & 1.00 & 1522.00 & 59.86 & 0.0000 \\
Age:Domain & 2.66 & 2.66 & 1.00 & 1552.00 & 5.65 & 0.0176 \\
\hline
\end{tabular}


Table 2: Anova Table $\gamma$

\begin{tabular}{lrrrrrr}
\hline GCMR & Sum Sq & Mean Sq & NumDF & DenDF & F value & Pr $(>\mathrm{F})$ \\
\hline Age & 2.03 & 2.03 & 1.00 & 1522.00 & 6.45 & 0.0112 \\
Domain & 0.00 & 0.00 & 1.00 & 1552.00 & 0.00 & 0.9981 \\
Item Set & 5.96 & 0.21 & 29.00 & 1522.00 & 0.65 & 0.9228 \\
Version Order & 0.27 & 0.27 & 1.00 & 1522.00 & 0.87 & 0.3512 \\
Age:Domain & 0.75 & 0.75 & 1.00 & 1552.00 & 2.36 & 0.1244 \\
\hline 2HT & & & & & & \\
\hline Age & 2.21 & 2.21 & 1.00 & 1522.00 & 6.57 & 0.0105 \\
Domain & 4.73 & 4.73 & 1.00 & 1552.00 & 14.07 & 0.0002 \\
Item Set & 7.93 & 0.27 & 29.00 & 1522.00 & 0.81 & 0.7482 \\
Version Order & 2.07 & 2.07 & 1.00 & 1522.00 & 6.16 & 0.0132 \\
Age:Domain & 1.75 & 1.75 & 1.00 & 1552.00 & 5.20 & 0.0228 \\
\hline
\end{tabular}


A
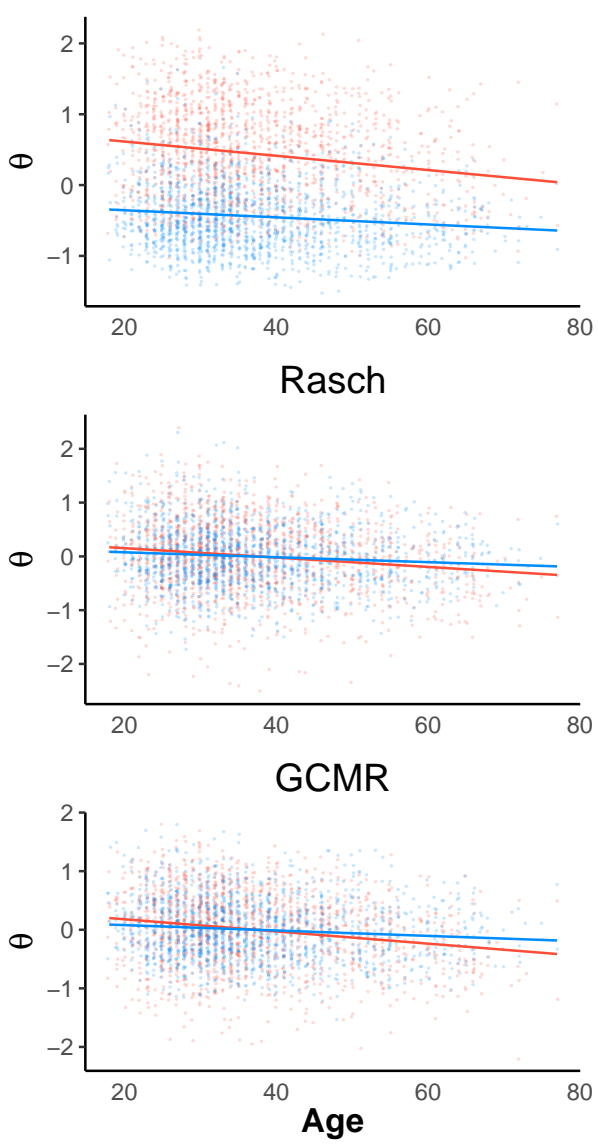

B

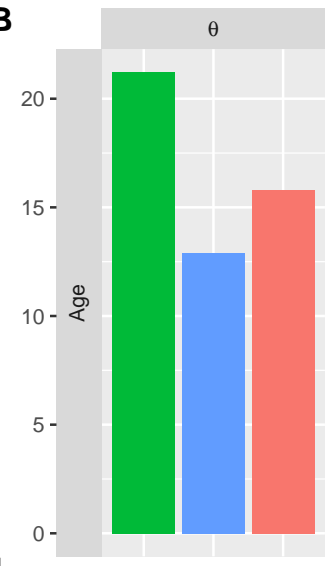

ᄂ

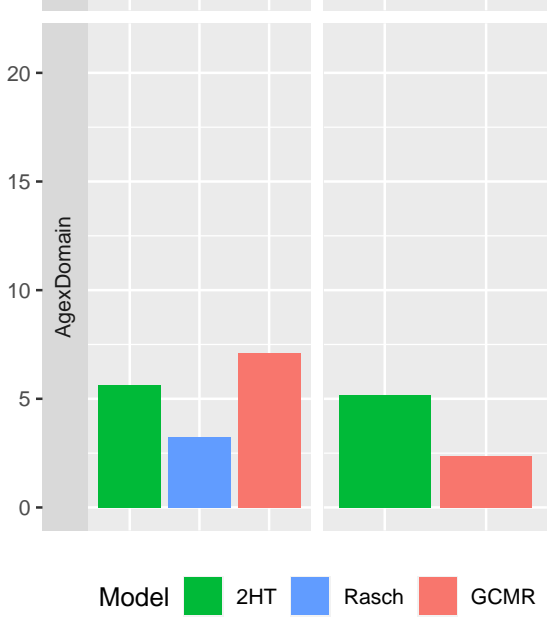

Figure 4: GCMR, Rasch and 2HT ability estimates across age.

A The plot shows the age $\mathrm{x}$ domain interaction effect slopes for ability $\theta$ in all three models. While the main effect of age was observed in all models, only GCMR and the $2 \mathrm{HT}$ model showed an age $\mathrm{x}$ domain interaction. B $\theta$ : While the $2 \mathrm{HT}$ shows strongest main effect of age, the GCMR shows strongest age $\mathrm{x}$ domain interaction effect. $\gamma$ : Both models show general bias increase with age, while only the $2 \mathrm{HT}$ shows an interaction with domain.

\subsection{Real data study II: CAT}

When presenting stimuli in the original administration order, trial-wise correlation of parameter estimates with full-sample estimates increased faster for the GCMR than the $2 \mathrm{HT}$ model, reaching $\rho=0.9$ after 50 trials for $\theta_{G C M R}$ and 56 trials for $\theta_{2 H T}$. However, when an adaptive adiministration order was used, only 26 trials where necessary for $\theta_{G C M R}$ to reach $\rho=0.9$. In order to compare trial-wise standard error of measurement $\left(S E_{\theta}\right)$ for $\theta_{G C M R}$ in the original versus 
adaptive administration order, we measured how many trials were required to obtain a high confidence of ability estimates with $S E_{\theta}$ smaller than 0.6. While 79 trials were necessary to reach this criterion when using a non-adaptive order, only 26 trials were necessary using CAT.

A

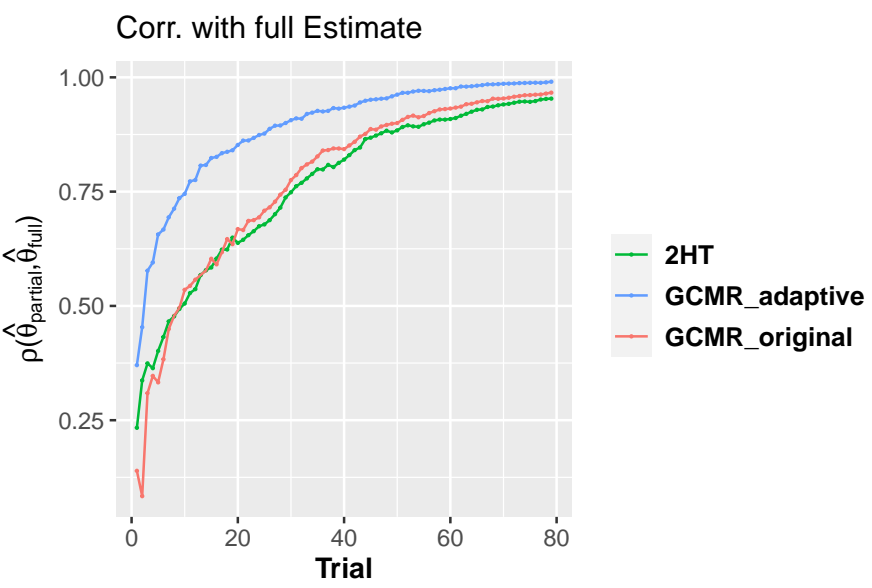

B

Measurement Error: $\theta_{\mathrm{GCMR}}$

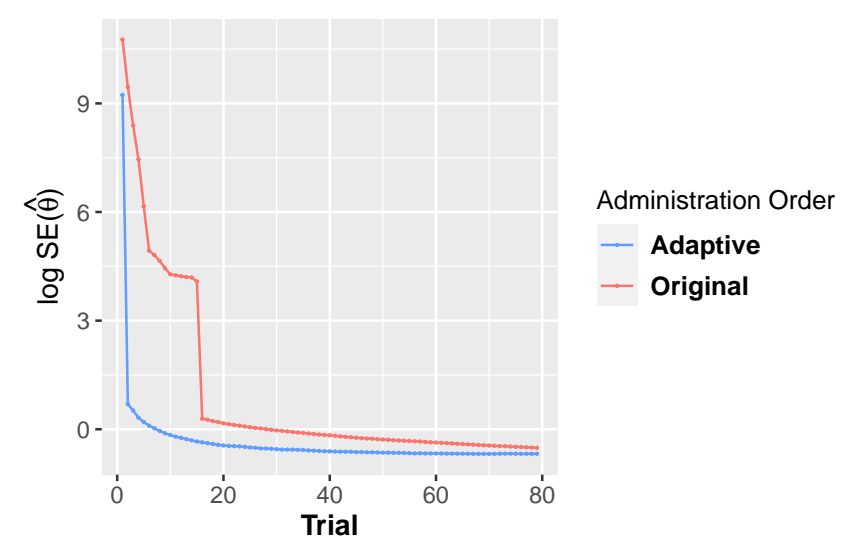

Figure 5: Trial-wise convergence of $\theta$ using original versus simulated adaptive item administration order.

A While convergence was observed to be similar for GCMR and the 2HT model in the original test administration order, convergence of abilities $\theta_{G C M R}$ was faster under an adaptive order. Standard error $\mathbf{B} S E_{\theta}$ decreases more rapidly in the adaptive than the original order, while both approaches converge in later trials. 


\section{Discussion}

In this paper, we introduce and validate a new General Condorcet Model for Recognition (GCMR) for dichotomous responses that combines aspects of the 2High-Threshold (2HT) for recognition memory and the Rasch model from Item Response Theory (Snodgrass and Corwin, 1988, Rasch, 1980). Formally, the proposed GCMR model belongs to a more general class of General Condorcet Models (Batchelder and Romney, 1988), with the restriction that the expected response is fixed by design rather than having to be estimated. The approach aims at overcoming the limitation of conventional recognition memory models that posit item homogeneity (e.g. $2 \mathrm{HT}, d^{\prime}$ ) by allowing for item difficulty to vary freely. GCMR is an IRT model, which separately parametrizes item difficulty and discrimination ability to enable ability estimation to be independent of the item set presented. Using both simulated and real data, we show that the proposed GCMR model outperforms both the Rasch an 2HT models in modeling old-new recognition memory data. We found favourable parameter recovery for the GCMR model and highlight cases in which ability estimates may be biased for both the Rasch and the $2 \mathrm{HT}$ model. Using real behavioural data from a mnemonic object-scene discrimination task, the GCMR also exhibited greater model evidence (using leave-one-out crossvalidation) compared to both the Rasch and 2HT model. Furthermore, face validity of GCMR was established by replicating previous findings of performance decline with higher age and a steeper decline in object compared to scene performance (Güsten et al., 2021). Finally, we demonstrated GCMR's potential for adaptive testing, which demonstrates that using GCMR we can overcome severe limitations from conventional cognitive non-IRT models.

The approach contributes to recent work combining psychometric with cognitive models from recognition memory (Thomas et al., 2018; DeCarlo, 2019), thereby drawing from advanced approaches to examine measurement precision as well as psychologically valid modeling of relevant latent variables. While Thomas et al. (2018) and DeCarlo (2019) both used SDT models as the basis of their cognitive models, to our knowledge GCMR is the first approach combining IRT with a recognition memory model from the MPT family aimed at assessing recognition memory. 2HT, as well as our proposed approach are particular instances of Binary MPTs (BMPT) called "Multitree MPTs" (Batchelder, 2010), which have the property to involve different within-subject trial types (e.g. targets versus foils). Many formal properties of these models as well as non-Bayesian estimation methods using the Expectation-Maximization (EM) algorithm have been previously discussed (Hu and Batchelder (1994)). GCMR might also be viewed as an example of what Batchelder (2010) referred to as "Rasch-BMPTs", in which the transition probabilities between branches are given by a Rasch model. While Karabatsos and Batchelder (2003) applied this approach to a particular MPT called the general Condorcet model, our GCMR model is a more tailored adaptation for recognition memory modelling to overcome limitations of more conventional $2 \mathrm{HT}$ approaches. As such, GCMR 
differs from the two above SDT-based models in important ways. For instance, Thomas et al. (2018) showed that a simple SDT model (d') can be extended to a compensatory multidimensional 2P IRT model (Reckase, 2009). This model effectively places bias and memory discrimination under a common process, whereby one process can entirely compensate for another. It is expected that under (infinitely) high bias the discrimination may become almost unmeasurable, leading to response patterns that are solely predicted by the trial type (target versus foil). As an example, under such high bias the correct response probability for foils would approximate zero. The GCMR is non-compensatory in that way, since the discrimination and guessing are modeled as independent processes. That is both guessing and perception process set a lower bound on performance. In the approach chosen by DeCarlo (2019) and similar to the wellknown 3PL IRT model, guessing or response bias is an item parameter while the GCMR defines it as a person-specific characteristic. We believe this to be a sensible and important feature of the present approach since guessing propensity has been shown to be influenced by clinical pathologies as well ageing, and might therefore serve as an important subject-specific behavioral marker in clinical assessment. However, GCMR represents but one effort to combine cognitive modeling with psychometric theory in order to assess recognition memory, and future empirical work is needed to determine which model is best suited to reflect the relevant latent processes.

The simulation study showed that parameter estimates of the $2 \mathrm{HT}$ and the Rasch model diverged strongly from GCMR ground truth under different manipulations of test difficulty, average response bias and data missingness, while the GCMR fared well in recovering ground truth parameters under these manipulations. One surprising observation was that a more biased guessing propensity did facilitate a more accurate estimation of ability, especially when the true ability ways low in comparison to test difficulty. One might speculate that under low ability the amount of guessing increases, making ability estimation harder since more prone to noise. However, when guessing is highly biased, the variance it adds to responses is low, which again seems to facilitate ability estimation. Comparatively, the GCMR performed well under these less favorable conditions. This might be because it accounts for more sources of response variability than the two models separately, that is response bias, ability, and item difficulty simultaneously. This finding is especially relevant when studying clinical or populations in old age, as these often differ in terms of memory abilities (Güsten et al., 2021; Berron et al., 2018, Stark and Stark, 2017; Reagh et al., 2016) and response bias (Huh et al., 2006) compared to healthy young adults.

Using cross-validation our results demonstrate that the GMCR shows better out-of-sample prediction for trial-by-trial response data than the $2 \mathrm{HT}$ or the Rasch model. This suggests that since GCMR models item heterogeneity it may be suitable for behavioral modeling of trial-by-trial performance. Apart from adaptive assessment it may be used to model subjective trial difficulty and could be combined with measures from other modalities (e.g. fMRI). We replicated deteriorative effects of age on mnemonic discrimination ability previ- 
ously reported by the authors (Güsten et al., 2021). One surprising finding is that the main effect of age was less significant for the GCMR than for the $2 \mathrm{HT}$ model. It may be that the effect is overestimated by the $2 \mathrm{HT}$. Another possibility is that part of GCMR's between-subject variance was modeled via the items. This might have arisen due to relatively weak item priors combined with few evaluations per item, and might have been biased by occasionally uneven item distributions across ability-related predictors such as age. One result showing the influence of free item parameters is that both the GCMR and Rasch model had weaker stimulus domain effects, being able to keep distributions for each domain around the prior mean, while the $2 \mathrm{HT}$ could not compensate for existing domain difficulty differences. This demonstrates the necessity to calibrate item parameters in a first step, when modeling subsamples independently. Unlike the 2HT model, GCMR is potentially able to model unbalanced item evaluations as long as their missingness is unrelated to ability, or predictors of ability (e.g. age). Even in such a case the GCMR can still be used if item parameters are calibrated on a suitable sample in advance. The domain-specific effect of age on ability was strongest for GCMR, which suggests that relevant variance regarding ability estimates was modeled. Domain-dependent age effects have been previously observed in other tasks assessing mnemonic discrimination (Stark and Stark, 2017; Reagh et al., 2016) and have been proposed to reflect distinct, domain-specific memory pathways (Ranganath and Ritchey, 2012) and their age-related dysfunction (Maass et al., 2019; Berron et al., 2019). The GCMR may therefore hold promise as a diagnostic tool in behavioral assessment of (domain-dependent) memory decline.

Finally, we demonstrated that GCMR might be useful for future studies to perform CAT in recognition memory tasks. However, unlike common IRT models focussing on a single person parameter we performed CAT on a model with two person parameters (ability and bias). To do so, a large set of items was calibrated and characterized using conventional item statistics from IRT. We were able to speed up convergence of ability estimation considerably by reducing the number of trials by $48 \%$ in order to reach $90 \%$ convergence. Real adaptive testing scenarios in future studies might prove to further speed up convergence. Here the algorithm could only choose items that were present in the actual data.

We also highlighted the benefits of obtaining trial-wise estimates of conditional measurement error and found that over trials the estimation error decreased significantly faster using an adaptive versus a non-adaptive item administration order. Based on these results CAT is promising in clinical research for its potential to make tests shorter and more efficient. As time-efficient test solutions are needed, this is particularly important for the growing field of mobile cognitive assessment. Regarding experimental work in recognition memory, the GCMR might contribute to the adaptive assessment of the well-studied cognitive variables memory ability and response bias in various populations.

It is important to mention some limitations and point out directions for future research. The complex structure of sparsity in our data set comes with costs when performing explorative analysis. Design sparsity prevented us from 
rigorous testing of further IRT assumptions such as conditional independence and dimensionality. It should be noted, however, that evidence in favour of two latent dimensions in recognition memory tasks has been found elsewhere (Thomas et al., 2018). Nevertheless, in future studies the GCMR might be tested on data from a more balanced design, where these assumptions can be easily checked. Furthermore, several sensible extensions to the model were not tested here. One the one hand, it is possible to include covariate information for each parameter in the form of a latent regression (Oravecz et al. 2013). For instance, one could directly model age as a person regressor and stimulus domain as an item regressor. Furthermore, instead of using a using a 2-stage approach, measuring population effects on ability with a separate LME, it might be efficient to specify these effects in context of a hierarchical formulation of the model (Oravecz et al. 2013 ). This would allow to preserve and propagate uncertainty information in the person parameters across individual and group level. Finally, the model in its current form assumes independence of ability and response bias, based on previous SDT studies showing that these variables can be manipulated separately (Swets, 2014). However, those variables might be associated empirically. Given that ability and bias are currently modeled using independent distributions, it is difficult to directly model their relation. One solution might be to model both person parameters as jointly normally distributed, which would allow easy estimation of their covariance (Oravecz et al. 2013).

In summary, we presented a GCM that accounts for varying item difficulty and is able to model trial-by-trial recognition memory performance better than the well-known $2 \mathrm{HT}$ model. Moreover, we were able to establish face validity by replicating earlier findings on recognition memory using the $2 \mathrm{HT}$ model. We demonstrated that the GCMR can be readily applied in CAT, leading to refined measurement estimates and increased test efficiency. We furthermore pointed out its great potential in the domain of clinical and mobile memory assessment. 


\section{Funding}

The work was funded by the German Research Foundation CRC 779 (TP A07).

\section{Appendix A}

Prior specification

\subsection{Rasch}

$$
\begin{array}{r}
\theta \sim N(0,1) \\
\beta \sim \mu_{\beta}+\sigma_{\beta} \cdot \alpha_{\beta} \\
\mu_{\beta} \sim \operatorname{cauchy}(0,5) \\
\sigma_{\beta} \sim \operatorname{cauchy}(0,5) \\
\alpha_{\beta} \sim N(0,1)
\end{array}
$$

\section{$4.2 \quad 2 \mathrm{HT}$}

$$
\begin{array}{r}
\theta \sim N(0,1) \\
\gamma \sim \operatorname{Beta}\left(\alpha_{\gamma}, \beta_{\gamma}\right) \\
\alpha_{\gamma} \geq 0 \\
\beta_{\gamma} \geq 0
\end{array}
$$

\subsection{GCMR}

$$
\begin{array}{r}
\theta \sim N(0,1) \\
\beta \sim \mu_{\beta}+\sigma_{\beta} \cdot \alpha_{\beta} \\
\gamma \sim \operatorname{Beta}\left(\alpha_{\gamma}, \beta_{\gamma}\right) \\
\mu_{\beta} \sim \operatorname{cauchy}(0,5) \\
\sigma_{\beta} \sim \operatorname{cauchy}(0,5) \\
\alpha_{\beta} \sim N(0,1) \\
\alpha_{\gamma} \geq 0 \\
\beta_{\gamma} \geq 0
\end{array}
$$

Notice that we used a hierarchical prior for beta. Furtermore, the parameterization was non-centered, in order to facilitate convergence of Hamiltonian Monte Carlo Sampling in Stan Betancourt and Girolami (2013). This is possible for Gaussian distributions, since

$$
\alpha \sim N(0,1) \Longrightarrow \mu+\sigma \cdot \alpha \sim N(\mu, \sigma)
$$




\section{Appendix B}

Item information function with respect to ability $\theta$

$$
I_{\theta}(\theta, \gamma)=\frac{\left[\frac{\partial}{\partial \theta} P(\theta, \gamma)\right]^{2}}{P(\theta, \gamma) \cdot(1-P(\theta, \gamma))}
$$

$P(\theta, \gamma)$ refers to the probability of a correct response provided above (eq. 5)

\section{Appendix C}
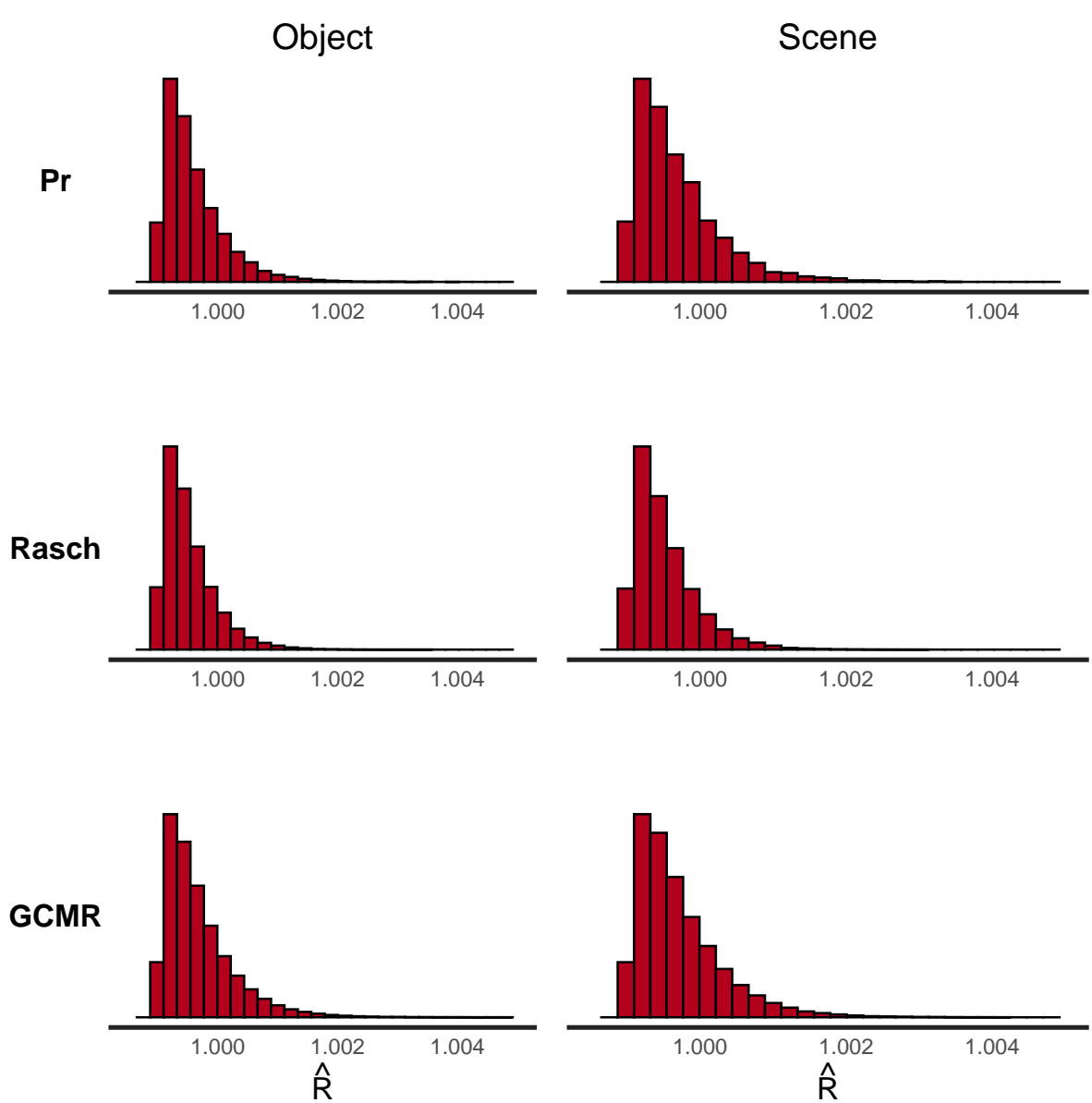

Figure 6: Model convergence.

All three models converged well for both domains. An $\hat{R}$-statistic of well below 1.1 indicates that parameter estimates were consistent across MCMC chains. 


\section{References}

Batchelder, W. H. (2010). Cognitive psychometrics: Using multinomial processing tree models as measurement tools. In Measuring psychological constructs: Advances in model-based approaches., pages 71-93. American Psychological Association.

Batchelder, W. H. and Romney, A. K. (1988). Test theory without an answer key. Psychometrika, 53(1):71-92.

Bates, D., Mächler, M., Bolker, B., and Walker, S. (2015). Fitting linear mixedeffects models using lme4. Journal of Statistical Software, 67(1):1-48.

Berron, D., Cardenas-Blanco, A., Bittner, D., Metzger, C. D., Spottke, A., Heneka, M. T., Fliessbach, K., Schneider, A., Teipel, S. J., Wagner, M., Speck, O., Jessen, F., and Düzel, E. (2019). Higher CSF tau levels are related to hippocampal hyperactivity and object mnemonic discrimination in older adults. The Journal of Neuroscience, 39(44):8788-8797.

Berron, D., Neumann, K., Maass, A., Schütze, H., Fliessbach, K., Kiven, V., Jessen, F., Sauvage, M., Kumaran, D., and Düzel, E. (2018). Age-related functional changes in domain-specific medial temporal lobe pathways. Neurobiology of Aging, 65:86-97.

Betancourt, M. J. and Girolami, M. (2013). Hamiltonian monte carlo for hierarchical models.

Beth, E. H., Budson, A. E., Waring, J. D., and Ally, B. A. (2009). Response bias for picture recognition in patients with alzheimer disease. Cognitive and Behavioral Neurology, 22(4):229-235.

Boeck, P. D. and Partchev, I. (2012). IRTrees: Tree-based item response models of the GLMM family. Journal of Statistical Software, 48(Code Snippet 1).

Brown, G. G., Thomas, M. L., and Patt, V. (2017). Parametric model measurement: reframing traditional measurement ideas in neuropsychological practice and research. The Clinical Neuropsychologist, 31(6-7):1047-1072.

DeCarlo, L. T. (2019). An item response model for true-false exams based on signal detection theory. Applied Psychological Measurement, 44(3):234-248.

Gavett, B. E. and Horwitz, J. E. (2011). Immediate list recall as a measure of short-term episodic memory: Insights from the serial position effect and item response theory. Archives of Clinical Neuropsychology, 27(2):125-135.

Gelman, A., Carlin, J. B., Stern, H. S., Dunson, D. B., Vehtari, A., and Rubin, D. B. (2013). Bayesian Data Analysis. Chapman and Hall/CRC.

Gelman, A. and Rubin, D. B. (1992). Inference from iterative simulation using multiple sequences. Statistical Science, 7(4):457-472. 
Gershon, R. C., Cook, K. F., Mungas, D., Manly, J. J., Slotkin, J., Beaumont, J. L., and Weintraub, S. (2014). Language measures of the NIH toolbox cognition battery. Journal of the International Neuropsychological Society, 20(6):642-651.

Gibbons, R. D., Kupfer, D. J., Frank, E., Lahey, B. B., George-Milford, B. A., Biernesser, C. L., Porta, G., Moore, T. L., Kim, J. B., and Brent, D. A. (2020). Computerized adaptive tests for rapid and accurate assessment of psychopathology dimensions in youth. Journal of the American Academy of Child Es Adolescent Psychiatry, 59(11):1264-1273.

Gibbons, R. D., Weiss, D. J., Pilkonis, P. A., Frank, E., Moore, T., Kim, J. B., and Kupfer, D. J. (2012). Development of a computerized adaptive test for depression. Archives of General Psychiatry, 69(11):1104.

Gibbons, R. D., Weiss, D. J., Pilkonis, P. A., Frank, E., Moore, T., Kim, J. B., and Kupfer, D. J. (2014). Development of the CAT-ANX: A computerized adaptive test for anxiety. American Journal of Psychiatry, 171(2):187-194.

Glas, C. A. W. and van der Linden, W. J. (2003). Computerized adaptive testing with item cloning. Applied Psychological Measurement, 27(4):247-261.

Güsten, J., Ziegler, G., Düzel, E., and Berron, D. (2021). Age impairs mnemonic discrimination of objects more than scenes: A web-based, large-scale approach across the lifespan. Cortex.

$\mathrm{Hu}, \mathrm{X}$. and Batchelder, W. H. (1994). The statistical analysis of general processing tree models with the EM algorithm. Psychometrika, 59(1):21-47.

Huh, T. J., Kramer, J. H., Gazzaley, A., and Delis, D. C. (2006). Response bias and aging on a recognition memory task. Journal of the International Neuropsychological Society, 12(1):1-7.

Jeon, M. and Boeck, P. D. (2015). A generalized item response tree model for psychological assessments. Behavior Research Methods, 48(3):1070-1085.

Kantner, J. and Lindsay, D. S. (2012). Response bias in recognition memory as a cognitive trait. Memory Cognition, 40:1163-1177.

Karabatsos, G. and Batchelder, W. H. (2003). Markov chain estimation for test theory without an answer key. Psychometrika, 68(3):373-389.

Maass, A., Berron, D., Harrison, T. M., Adams, J. N., Joie, R. L., Baker, S., Mellinger, T., Bell, R. K., Swinnerton, K., Inglis, B., Rabinovici, G. D., Düzel, E., and Jagust, W. J. (2019). Alzheimer's pathology targets distinct memory networks in the ageing brain. Brain, 142(8):2492-2509.

Macmillan, N. A. and Creelman, C. D. (2004). Detection Theory. Psychology Press. 
Magis, D. and Raîche, G. (2012). Random generation of response patterns under computerized adaptive testing with the R package catR. Journal of Statistical Software, 48(8):1-31.

Morcom, A. M., Good, C. D., Frackowiak, R. S. J., and Rugg, M. D. (2003). Age effects on the neural correlates of successful memory encoding. Brain, $126(1): 213-229$.

Olino, T. M., Yu, L., Klein, D. N., Rohde, P., Seeley, J. R., Pilkonis, P. A., and Lewinsohn, P. M. (2012). Measuring depression using item response theory: an examination of three measures of depressive symptomatology. International Journal of Methods in Psychiatric Research, 21(1):76-85.

Oravecz, Z., Anders, R., and Batchelder, W. H. (2013). Hierarchical bayesian modeling for test theory without an answer key. Psychometrika, 80(2):341364.

Pedraza, O., Sachs, B. C., Ferman, T. J., Rush, B. K., and Lucas, J. A. (2011). Difficulty and discrimination parameters of boston naming test items in a consecutive clinical series. Archives of Clinical Neuropsychology, 26(5):434444.

Ranganath, C. and Ritchey, M. (2012). Two cortical systems for memory-guided behaviour. Nature Reviews Neuroscience, 13(10):713-726.

Ranger, J. and Ortner, T. M. (2011). Assessing personality traits through response latencies using item response theory. Educational and Psychological Measurement, 71(2):389-406.

Rasch, G. (1980). Probabilistic models for some intelligence and attainment tests. University of Chicago Press, Chicago.

Reagh, Z. M., Ho, H. D., Leal, S. L., Noche, J. A., Chun, A., Murray, E. A., and Yassa, M. A. (2016). Greater loss of object than spatial mnemonic discrimination in aged adults. Hippocampus, 26(4):417-422.

Reckase, M. D. (2009). Multidimensional Item Response Theory. SpringerVerlag GmbH.

Reise, S. P. and Henson, J. M. (2000). Computerization and adaptive administration of the NEO PI-r. Assessment, 7(4):347-364.

Samejima, F. (1969). Estimation of latent ability using a response pattern of graded scores. Psychometrika, 34(S1):1-97.

Smith, A. B., Rush, R., Fallowfield, L. J., Velikova, G., and Sharpe, M. (2008). Rasch fit statistics and sample size considerations for polytomous data. $B M C$ Medical Research Methodology, 8(1). 
Snodgrass, J. G. and Corwin, J. (1988). Pragmatics of measuring recognition memory: Applications to dementia and amnesia. Journal of Experimental Psychology: General, 117(1):34-50.

Spence, R., Owens, M., and Goodyer, I. (2012). Item response theory and validity of the NEO-FFI in adolescents. Personality and Individual Differences, $53(6): 801-807$.

Stan Development Team (2020). RStan: the R interface to Stan. R package version 2.21.2.

Stark, S. M. and Stark, C. E. (2017). Age-related deficits in the mnemonic similarity task for objects and scenes. Behavioural Brain Research, 333:109117.

Sunderland, M., Batterham, P., Carragher, N., Calear, A., and Slade, T. (2017). Developing and validating a computerized adaptive test to measure broad and specific factors of internalizing in a community sample. Assessment, 26(6):1030-1045.

Swets, J. (2014). Signal detection theory and ROC analysis in psychology and diagnostics : collected papers. Psychology Press, New York, New York Hove, England.

Thomas, M. L. (2010). The value of item response theory in clinical assessment: A review. Assessment, 18(3):291-307.

Thomas, M. L. (2019). Advances in applications of item response theory to clinical assessment. Psychological Assessment, 31(12):1442-1455.

Thomas, M. L., Brown, G. G., Gur, R. C., Moore, T. M., Patt, V. M., Risbrough, V. B., and Baker, D. G. (2018). A signal detection-item response theory model for evaluating neuropsychological measures. Journal of Clinical and Experimental Neuropsychology, 40(8):745-760.

Vehtari, A., Gabry, J., Magnusson, M., Yao, Y., and Gelman, A. (2019). loo: Efficient leave-one-out cross-validation and waic for bayesian models. $\mathrm{R}$ package version 2.2.0.

Walter, O. B., Becker, J., Bjorner, J. B., Fliege, H., Klapp, B. F., and Rose, M. (2007). Development and evaluation of a computer adaptive test for 'anxiety' (anxiety-CAT). Quality of Life Research, 16(S1):143-155.

Weintraub, S., Dikmen, S. S., Heaton, R. K., Tulsky, D. S., Zelazo, P. D., Bauer, P. J., Carlozzi, N. E., Slotkin, J., Blitz, D., Wallner-Allen, K., Fox, N. A., Beaumont, J. L., Mungas, D., Nowinski, C. J., Richler, J., Deocampo, J. A., Anderson, J. E., Manly, J. J., Borosh, B., Havlik, R., Conway, K., Edwards, E., Freund, L., King, J. W., Moy, C., Witt, E., and Gershon, R. C. (2013). Cognition assessment using the NIH toolbox. Neurology, 80(Issue 11, Supplement 3):S54-S64. 
Yao, Y., Vehtari, A., Simpson, D., and Gelman, A. (2017). Using stacking to average bayesian predictive distributions. Bayesian Analysis. 